\title{
Impact of MLC properties and IMRT technique in meningioma and head-and-neck treatments
}

\author{
Steffi Kantz ${ }^{1 *}$, Matthias Söhn ${ }^{1}$, Almut Troeller ${ }^{1,2}$, Michael Reiner ${ }^{1}$, Helmut Weingandt ${ }^{1}$, Markus Alber ${ }^{3}$, \\ Claus Belka ${ }^{1}$ and Ute Ganswindt ${ }^{1}$
}

\begin{abstract}
Purpose: The impact of multileaf collimator (MLC) design and IMRT technique on plan quality and delivery improvements for head-and-neck and meningioma patients is compared in a planning study.

Material and methods: Ten previously treated patients ( 5 head-and-neck, 5 meningioma) were re-planned for step-and-shoot IMRT (ssIMRT), sliding window IMRT (dMLC) and VMAT using the MLCi2 without (-) and with (+) interdigitation and the Agility-MLC attached to an Elekta 6MV linac. This results in nine plans per patient. Consistent patient individual optimization parameters are used. Plans are generated using the research tool Hyperion V2.4 (equivalent to Elekta Monaco 3.2) with hard constraints for critical structures and objectives for target structures. For VMAT plans, the improved segment shape optimization is used.

Critical structures are evaluated based on QUANTEC criteria. PTV coverage is compared by EUD, $\mathrm{D}_{\text {mean, }}$, homogeneity and conformity. Additionally, MU/plan, treatment times and number of segments are evaluated.
\end{abstract}

Results: As constrained optimization is used, all plans fulfill the hard constraints. Doses to critical structures do not differ more than $1 G y$ between the nine generated plans for each patient. Only larynx, parotids and eyes differ up to $1.5 \mathrm{~Gy}\left(\mathrm{D}_{\text {mean }}\right.$ or $\left.\mathrm{D}_{\max }\right)$ or $7 \%$ (volume-constraint) due to (1) increased scatter, (2) not avoiding structures when using the full range of gantry rotation and (3) improved leaf sequencing with advanced segment shape optimization for VMAT plans. EUD, D mean, homogeneity and conformity are improved using the Agility-MLC. However, PTV coverage is more affected by technique. MU increase with the use of $\mathrm{AMLC}$ and VMAT, while the MU are reduced by using the Agility-MLC. Fastest treatments are always achieved using Agility-MLC, especially in combination with VMAT.

Conclusion: Fastest treatments with the best PTV coverage are found for VMAT plans with Agility-MLC, achieving the same sparing of healthy tissue compared to the other combinations of ssIMRT, dMLC and VMAT with either MLCi2 ${ }^{-/+}$ or Agility.

Keywords: MLC properties, IMRT, VMAT

\section{Introduction}

The major vendors of clinically used linear accelerators (linacs) re-designed their multi-leaf-collimators (MLCs) over time as importance of the leaf width, transmission, maximum leaf speed and leaf positioning accuracy as well as interdigitation capabilities was investigated in the literature.

Burmeister et al. [1] and Wu et al. [2] demonstrated that leaf width has only small impact on the plan quality

\footnotetext{
*Correspondence: Steffi.Kantz@med.uni-muenchen.de

'Department of Radiation Oncology, Ludwig-Maximilians-University, Munich, Germany

Full list of author information is available at the end of the article
}

for large PTVs. Benefits of smaller leaf width and leaf penumbra are predominantly encountered in cases of small lesions and complex PTV or organs at risks (OAR) [3-6]. Furthermore, smaller leaves may reduce radiation to surrounding tissue by up to $5 \%$ for the 70 and $50 \%$ isodose [4]. Bortfeld et al. [7] showed that the optimal leaf width is $1.5-1.8 \mathrm{~mm}$ when neglecting transmission.

On the other hand, Topolniak et al. [5] showed transmission to be one of the most important parameters towards higher quality plans. As lower transmission means lower dose to surrounding tissues, either less dose to healthy tissue or higher modulation degrees become feasible.

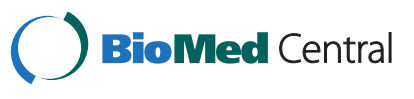


The importance of leaf positioning accuracy was demonstrated for various MLC designs, showing that leaf positioning errors need to be considered particularly when the leaves are moving at maximum speed and/or for highly modulated MLC patterns [8-11]. Furthermore, Low [8] and Vorwerk [12] proved the dependency of treatment time on leaf speed limitation. Thereby, not only the positioning accuracy, but also the velocity of the leaves is important for high quality plan delivery.

Concerning MLC interdigitation, literature reveals contradictory findings. Webb [13] describes this feature as favorable and Tacke et al. [14] show faster delivery times for complex step-and-shoot IMRT treatment plans (head-and neck, prostate including lymph nodes). For VMAT treatments, Van Kesteren et al. [15] show no improvement for prostate and rectum treatment plans whereas Lafond et al. [16] report at least improvements in delivery efficiency.

While Varian (Varian Medical Systems, Palo Alto, CA, USA) was the first company to introduce a MLC with a leaf width of $5 \mathrm{~mm}$ for large fields $\left(40 \times 40 \mathrm{~cm}^{2}\right)-$ at least for the inner $20 \mathrm{~cm}$-, Siemens (Siemens Medical Solutions, Germany) was then the first to provide a MLC with $5 \mathrm{~mm}$ leaf width over the whole $40 \times 40 \mathrm{~cm}^{2}$ field [14]. Besides the Beam Modulator ${ }^{\mathrm{TM}}$ MLC (4 mm leaves over a maximal field size of $21 \times 16 \mathrm{~cm}^{2}$ ) and the MLCi2 $\left(10 \mathrm{~mm}\right.$ leaves over the whole field size of $40 \times 40 \mathrm{~cm}^{2}$ ), Elekta (Elekta AB, Stockholm, Sweden) also introduced a MLC with $5 \mathrm{~mm}$ leaves over a $40 \times 40 \mathrm{~cm}^{2}$ field size (Agility $^{\mathrm{Tm}}$-MLC). Compared to the Beam-ModulatorMLC and the MLCi2, the Agility-MLC has lower transmission, as well as improved tongue-and-grove-effect and penumbra [17].

Numerous studies exist that evaluate the impact of some specific MLC design parameters on specific delivery techniques or the impact of different delivery techniques on certain treatment sites based on miscellaneous physical and clinical parameters of the dose distribution. As the various studies used diverse treatment planning approaches, optimization and dose calculation algorithms, the relationship of MLC design, delivery technique, optimization approach and treatment site is hard to obtain. To facilitate conclusions about the influence of different MLC design parameters with regard to different IMRT techniques for complex treatment volumes, this work presents a consistent planning study comparing three different MLC designs used with three different IMRT techniques for two treatment sites, by using a single treatment planning system.

The influence on clinical relevant parameters (target coverage, dose to critical structures, monitor units per plan and treatment times) is shown for different MLC designs by means of the Elekta MLCi2-MLC (without $(-)$ and with (+) interdigitation) and the Elekta Agility-
MLC and for different IMRT techniques by means of step-and-shoot IMRT (ssIMRT), dynamic-sliding window IMRT (dMLC) and volumetric modulated arc therapy (VMAT). For this comparison, plans were generated for meningioma and head-and-neck cases, using the treatment planning software Hyperion V2.4 (University of Tübingen, Germany, research version of Elekta MONACO 3.2) that employs constrained optimization and a Monte Carlo dose algorithm.

\section{Material and methods}

A planning study was performed for five head-and-neck $(\mathrm{HN})$ and five meningioma (MG) cases. For each case, nine plans were generated: three step-and-shoot IMRT (ssIMRT), three dynamic-sliding-window-IMRT (dMLC) and three volumetric modulated arc therapy (VMAT) plans, with each technique using the MLCi2-MLC with and without interdigitation $\left(\mathrm{MLCi}^{+}, \mathrm{MLCi} 2^{-}\right)$and the Agility-MLC. All plans were generated for an Elekta $6 \mathrm{MV}$ linac.

\section{Treatment planning system}

Hyperion V2.4 (University Tübingen, Germany, research version of Elekta MONACO 3.2) was used as treatment planning system [18-20]. It uses constrained optimization, i.e. at all stages of optimization all dose-limiting constraints are ensured to be strictly fulfilled. Hence, in constrained optimization, PTV coverage is an objective that will be fulfilled only, if the dose-limiting constraints allow for that. The functions applied in the optimization may not only be physical constraints (e.g. dose-volume-constraints, quadratic overdose, etc.) but also functions modelling the biological effect of radiation to different tissues (e.g. serial or parallel equivalent uniform dose, EUD) as described by Alber et al. [21, 22] The optimization is implemented as a two-step approach: First, the fluence matrix is optimized using an advanced pencil beam algorithm [23, 24]. Second, the fluence matrix is segmented into an initial-guess MLCsequence and subsequently optimized employing a segment shape and weight optimization [25]. Final dose calculation is performed with the XVMC Monte-Carlo dose engine [26].

VMAT optimization includes the use of the recently introduced advanced segment shape optimization, as part of the second stage optimization algorithm. During the first optimization stage, VMAT fluences are obtained on equidistant, user-defined gantry angles (15 during this study). Sequencing translates the fluence maps to deliverable segments at all available angles as sets of control points with definition of gantry angle, dose as MU for the segment and position for every leaf and jaw. Due to the rotation around the patient, differences in the OAR-to-PTV projection occur between the 15 equidistant gantry sampling points used during the fluence 
optimization and the continuous gantry positions used after the sequencing step. These are accounted for by the advanced segment shape optimization by checking every MLC position for each segment with regard to its influence on the OAR constraints and the PTV objectives. Thereby each leaf is opened or closed iteratively to find its optimal position.

\section{MLC properties}

The main properties of a MLC are the leaf width, maximum speed and minimal gap between opposing leaves as well as interdigitation capabilities. Table 1 shows all relevant differences between the MLCi2-MLC and the Agility-MLC. Generally, the smaller the leaf width, the better the field shape can be conformed with respect to the PTV and OARs. This potentially results in better OAR-sparing while the PTV coverage is maintained. A minimal leaf gap between two opposing leaves needs to be maintained for dynamic techniques to prevent colliding leaves. On the other hand, radiation passes through this gap and causes unwanted dose to tissue and reduces thereby some degree of freedom in the optimization process. Therefore, a small leaf gap with low radiation passing through is wanted. Another possibility would be backup jaws behind the leaves, under which leaf gaps could be parked. This could, however, introduce longer leaf travel paths and thereby increase treatment times and additionally require interdigitation capabilities. Furthermore, the capabilities of the backup jaw to reduce inter-leaf transmission vanishes for dynamic techniques like AMLC and VMAT as the backup jaws can only keep up with the most retracted leaf. Hence, leaves with a larger height can reduce the total transmission radiation. Concerning interdigitation, MLCs with such capabilities basically allow for independent placement of leaf positions for all leaf pairs. Generally, this facilitates more complex segment shapes as well as a larger search space of segment shapes for the sequencing algorithm to find an optimal plan. As example, interdigitation offers the possibility to treat multiple small field openings together within one segment, which may help to reduce the number of segments for a given degree of modulation of a treatment plan as compared to a non-interdigitating MLC. This potentially reduces the treatment time and

Table 1 MLC parameters for MLCi2 and Agility-MLC

\begin{tabular}{lll}
\hline MLC parameter & MLCi2 & Agility \\
\hline Leaf width & $10 \mathrm{~mm}$ & $5 \mathrm{~mm}$ \\
Leaf speed & $20 \mathrm{~mm} / \mathrm{s}$ & $65 \mathrm{~mm} / \mathrm{s}$ \\
& & incl. leaf guide \\
Min. leaf gap & $5 \mathrm{~mm}$ & $3 \mathrm{~mm}$ \\
Interdigitation & $-/+$ & + \\
Backup jaws & yes & no \\
\hline
\end{tabular}

MU, especially for larger and complex PTVs. The maximum speed of the leaves contributes to several properties. If the range of available speed is larger, faster plan delivery with a higher modulation degree is possible; also potentially reducing $\mathrm{MU}$ and scatter radiation.

Thus, different parameters of the MLC can increase or reduce the degrees of freedom for the optimizer and deliverability to a different amount. The MLCi2 -/+ interdigitation and the Agility-MLC show differences in these parameters and capabilities (Table 1), and this study aims to investigate their contribution to different plans and treatment sites, which might be different and not easily foreseen a priori.

\section{Patients}

Head and neck cases (HN), being considered challenging cases of current clinical practice [5], [27], and meningioma (MG) are chosen for the evaluation of the impact of MLC design and IMRT technique to the plan quality and potential benefits for the patient due to scatter radiation (MU) and treatment times. Each group includes five cases of previously treated patients. For $\mathrm{HN}$, target volume delineation and dose prescription are according to the ACCRA study [28] requirements with two or three dose levels (61.6/50.4 Gy or 61.6/56/50.4 Gy), prescribed as SIB-technique in 28 fractions. MG were treated with 54 Gy in 30 fractions. The MG PTV includes the GTV based on MR and PET imaging with a margin of not more than $5 \mathrm{~mm}$. Additional PRVmargins ( 3 to $5 \mathrm{~mm}$ ) to critical structures (e.g. optical nerves) were applied to improve OAR sparing. Complete characteristics of the included datasets are shown in Tables 2 and 3.

\section{Optimization parameters}

To access differences due to the IMRT-technique and the used MLC, all generated plans of the respective case are optimized applying the same patient-individual optimization parameters, i.e. same constrained optimization functions for OAR and PTV objectives with the related parameters, same gantry and collimator angles for ssIMRT and dMLC, as well as same Monte Carlo parameters (beam model of the radiation sources). Previous studies [29] show that VMAT plans generated using the Agility-MLC are superior in terms of higher PTV coverage and homogeneity without higher doses to organs at risk (OAR). Therefore, the applied optimization parameters are obtained from the VMAT ${ }^{\text {Agility }}$ plan separately for each case. To emphasize differences in the delivery of the techniques and the MLC properties, the applied constraints in this study are not those that were chosen for the clinical treatment plan generation. Instead, dose-limiting constraints are determined individually on a case-specific basis as low as realizable such that the PTV coverage is just not 
Table 2 Patient characteristics

\begin{tabular}{clc}
\hline Meningioma & Location & PTV volume $\left[\mathrm{cm}^{3}\right]$ \\
WHO I & & \\
\hline MG1 & os sphenoidale right & 42 \\
M2G & frontobasal right, intra-/supra-sellar & 107 \\
MG3 & orbital/parasellar right, frontal & 173 \\
MG4 & temporal right & 207 \\
MG5 & parasellar & 80 \\
\hline
\end{tabular}

affected for the VMAT ${ }^{\text {Agility }}$ plan when using two or three full $360^{\circ}$ arcs. These constraints are then used for all other plans of the respective case. Gantry angles for ssIMRT and dMLC are obtained from the ssIMRT ${ }^{\mathrm{MLCi2}-}$ plan on the basis of the clinically used gantry angles.

Using constraint optimization, OARs will end up fulfilling the dose-limiting constraints (mainly) to the same extent and differences between the plans will be shown in terms of PTV coverage.

\section{Plan evaluation}

Plans are evaluated using dose-volume-histogram (DVH) analysis and clinically important plan parameters. OAR exposure is evaluated based on QUANTEC criteria. For $\mathrm{HN}, \mathrm{D}_{\max }$ (dose to $1 \%$ of the volume) for spinal cord, brain stem, plexus (brachialis ipsilateral, contralateral) and mandibula, $D_{\text {mean }}$ and $V_{30 G y}$ of the contralateral parotid gland as well as $D_{\text {mean }}$ and $V_{50 G y}$ of the larynx are evaluated. For MG, OARs are evaluated by means of $\mathrm{D}_{\max }$ (dose to $1 \%$ of the volume) for brainstem, chiasm, optic nerves, eyes, lenses and brain. Additionally, mean doses for the eyes and $\mathrm{V}_{12 \mathrm{~Gy}}$ of the brain are evaluated.

PTV coverage analysis uses an equivalent uniform dose- (EUD-) definition based on the Poisson model [18], $\mathrm{D}_{\text {mean }}$, homogeneity (according to ICRU 83, formula 1), and conformity (suggested by Paddick [30], formula 2).

$$
H I_{\text {ICRU }}=\frac{D_{2} \%-D_{98} \%}{D_{50 \%}}
$$

The homogeneity index (HI) will tend to zero, the better the homogeneity of the plan is.
The conformity index (CI) accounts for prescribed dose outside the PTV and underdosage of the PTV, such that it equals 1 , only if the prescription isodose is surrounding the PTV completely without extending into normal tissue. Otherwise CI is smaller. The CI is calculated for the $95 \%$ - and $100 \%$-isodose:

$$
C I_{x \%}=\frac{\left(V_{D(P T V)=x \%}\right)^{2}}{\left(V_{P T V} * V_{D(\text { bod } y)=x \%}\right)}
$$

Number of segments per plan, MU (as equivalent to the modulation degree) and estimated treatment times are compared as clinically important parameters.

As the PTV for each patient has quite a wide range in volume, location and proximity to OARs, the evaluation is done patient-wise. The following comparisons are made:

$\alpha)$ To have a general overview, each plan is compared to VMAT Agility.

B) In order to distinguish differences caused by the treatment technique independently of which MLC was used, each technique was compared to the corresponding VMAT plan using the same MLC. Therefore three underlying comparisons were made, which evaluate the influence of using sIMRT, dMLC or VMAT together with MLCi2 $2^{-}, \mathrm{MLCi}^{+}$ and Agility. In the results Tables 4 and 5, comparison of sIMRT and dMLC to VMAT using the MLCi2- would correspond to comparing column A/D vs. G $\left(\beta^{\mathrm{MLCi} 2-}\right)$, while comparing sIMRT and dMLC to VMAT using MLCi2+ and Agility corresponds to $\mathrm{B} / \mathrm{E} v s . \mathrm{H}\left(\beta^{\mathrm{MLCi} 2+}\right)$ and $\mathrm{C} / \mathrm{F} v s . \mathrm{I}\left(\beta^{\text {Agility }}\right)$, respectively.

\begin{tabular}{|c|c|c|c|c|c|}
\hline \multicolumn{3}{|l|}{ HNSCC } & \multicolumn{3}{|c|}{ PTV volume $\left[\mathrm{cm}^{3}\right]$} \\
\hline Oropharynx & stage & location & $\mathrm{PTV}_{61.6 \mathrm{~Gy}}$ & $\mathrm{PTV}_{56 \mathrm{~Gy}}$ & $\mathrm{PTV}_{50.4 \mathrm{~Gy}}$ \\
\hline $\mathrm{HN1}$ & pT1 pN2b M0 L0 V0 & right & 132 & & 834 \\
\hline $\mathrm{HN} 2$ & pT2 pN2b M0 L1 V1 & left & 145 & 296 & 1240 \\
\hline HN3 & pT3 pN2b M0 L1 V1 & right & 422 & 525 & 1006 \\
\hline HN4 & pT2 pN2b MO LO V0 & right & 133 & 267 & 823 \\
\hline HN5 & PT3 pNO MO LO VO & left & 166 & & 659 \\
\hline
\end{tabular}

Table 3 Patient characteristics 
Table 4 Results for nine plan groups (3 techniques $+3 \mathrm{MLC}$ ) for the evaluated parameters for the head-and-neck cases (average and standard deviation for five patients): QUANTEC criteria where applicable

\begin{tabular}{|c|c|c|c|c|c|c|c|c|c|c|c|c|c|c|c|c|c|c|c|c|}
\hline \multicolumn{2}{|c|}{ Head and neck } & \multirow[t]{2}{*}{ Criteria } & \multicolumn{6}{|c|}{ Step-and-shoot IMRT } & \multicolumn{6}{|l|}{ dmlc IMRT } & \multicolumn{6}{|l|}{ VMAT } \\
\hline & & & \multicolumn{2}{|l|}{$\overline{\mathrm{MLCi}^{-}}$} & \multicolumn{2}{|l|}{$\mathrm{MLCi}^{+}$} & \multicolumn{2}{|l|}{ Agility } & \multicolumn{2}{|l|}{$\overline{\mathrm{MLCi}^{-}}$} & \multicolumn{2}{|l|}{$\mathrm{MLCi}^{+}$} & \multicolumn{2}{|l|}{ Agility } & \multicolumn{2}{|l|}{$\overline{\mathrm{MLCi}^{-}}$} & \multicolumn{2}{|l|}{$\mathrm{MLCi}^{+}$} & \multicolumn{2}{|l|}{ Agility } \\
\hline Segments $^{a}$ & & - & $97.6 \pm 18.3$ & + & $85.8 \pm 14.0$ & + & $78.2 \pm 11.7$ & & $16930 \pm 2.6$ & $=$ & $16935 \pm 2.7$ & $=$ & $171.2 \pm 1.1$ & & $338.0 \pm 56.7$ & $=$ & $353.8 \pm 76.6$ & $=$ & $375.0 \pm 57.5$ & 1 \\
\hline MU & & - & $86.1 \pm 150.5$ & $=$ & $742.2 \pm 109.3$ & $<$ & $657.2 \pm 95.2$ & $<$ & $777.4 \pm 65.8$ & $<$ & $803.4 \pm 75.9$ & $<$ & $803.9 \pm 87.1$ & $=$ & $959.5 \pm 98.4$ & + & $983.1 \pm 123.1$ & + & $893.8 \pm 96.3$ & 2 \\
\hline $\begin{array}{l}\text { Estimated } \\
\text { delivery } \\
\text { time [s] }\end{array}$ & & - & $607.4 \pm 92.0$ & + & $571.1 \pm 71.2$ & + & $447.2 \pm 55.7$ & + & $421.2 \pm 30.7$ & + & $420.3 \pm 25.0$ & + & $323.2 \pm 31.4$ & + & $368.3 \pm 28.6$ & + & $361.7 \pm 44.7$ & + & $224.4 \pm 23.1$ & 3 \\
\hline \multirow[t]{5}{*}{$\mathrm{PTV}_{50} 4 \mathrm{~Gy}$} & EUD & $50.4 \Uparrow$ & $47.7 \pm 0.2$ & $<$ & $47.7 \pm 0.3$ & $<$ & $47.9 \pm 0.5$ & $<$ & $48.8 \pm 0.6$ & $<$ & $49.1 \pm 0.6$ & $<$ & $49.0 \pm 0.4$ & $<$ & $49.5 \pm 0.8$ & $<$ & $49.5 \pm 0.7$ & $<$ & $50.1 \pm 0.5$ & 4 \\
\hline & $D_{\text {mean }}$ & $\Uparrow$ & $52.0 \pm 1.6$ & $<$ & $52.1 \pm 1.5$ & $<$ & $52.1 \pm 1.5$ & $<$ & $52.9 \pm 1.6$ & $<$ & $52.9 \pm 1.6$ & $<$ & $53.0 \pm 1.5$ & $<$ & $53.1 \pm 1.8$ & $<$ & $53.1 \pm 1.7$ & $<$ & $53.3 \pm 1.7$ & 5 \\
\hline & $H_{I C R U}$ & $\Downarrow$ & $0.436 \pm 0.041$ & + & $0.435 \pm 0.041$ & + & $0.431 \pm 0.037$ & + & $0.408 \pm 0.031$ & + & $0.399 \pm 0.032$ & + & $0.404 \pm 0.036$ & + & $0.380 \pm 0.032$ & + & $0.382 \pm 0.031$ & + & $0.373 \pm 0.032$ & 6 \\
\hline & $\mathrm{Cl}_{100 \%}$ & $\Uparrow$ & $0.420 \pm 0.098$ & $<$ & $0.433 \pm 0.090$ & $<$ & $0.429 \pm 0.088$ & $<$ & $0.495 \pm 0.075$ & $<$ & $0.507 \pm 0.071$ & $<$ & $0.522 \pm 0.066$ & $=$ & $0.523 \pm 0.106$ & $<$ & $0.521 \pm 0.088$ & $<$ & $0.566 \pm 0.091$ & 7 \\
\hline & $\mathrm{Cl}_{95} \%$ & $\Uparrow$ & $0.617 \pm 0.040$ & $<$ & $0.629 \pm 0.038$ & $<$ & $0.634 \pm 0.031$ & $<$ & $0.678 \pm 0.027$ & $=$ & $0.684 \pm 0.028$ & $=$ & $0689 \pm 0.026$ & $=$ & $0.692 \pm 0.034$ & $<$ & $0.691 \pm 0.031$ & $<$ & $0.711 \pm 0.033$ & 8 \\
\hline \multirow[t]{5}{*}{ PTV 61.6 Gy } & EUD & $61.6 \Uparrow$ & $58.4 \pm 1.2$ & $<$ & $58.6 \pm 1.0$ & $<$ & $58.9 \pm 1.3$ & $<$ & $59.9 \pm 0.8$ & $<$ & $60.1 \pm 0.9$ & $<$ & $60.3 \pm 0.9$ & $=$ & $60.3 \pm 1.0$ & $<$ & $60.2 \pm 1.0$ & $<$ & $60.7 \pm 0.9$ & 9 \\
\hline & $D_{\text {mean }}$ & $\Uparrow$ & $60.3 \pm 0.7$ & $<$ & $60.4 \pm 0.7$ & $<$ & $60.5 \pm 0.8$ & $<$ & $61.2 \pm 0.5$ & $<$ & $61.3 \pm 0.6$ & $<$ & $61.4 \pm 0.6$ & $=$ & $61.4 \pm 0.7$ & $<$ & $61.3 \pm 0.7$ & $<$ & $61.6 \pm 0.6$ & 10 \\
\hline & $\mathrm{HI}_{\mathrm{ICRU}}$ & $\Downarrow$ & $0.175 \pm 0.035$ & + & $0.172 \pm 0.036$ & + & $0.165 \pm 0.040$ & + & $0.140 \pm 0.028$ & + & $0.135 \pm 0.030$ & + & $0.127 \pm 0.033$ & $=$ & $0.128 \pm 0.025$ & + & $0.132 \pm 0.028$ & + & $0.120 \pm 0.027$ & 11 \\
\hline & $\mathrm{Cl}_{100} \%$ & $\Uparrow$ & $0.322 \pm 0.084$ & $<$ & $0.326 \pm 0.098$ & $<$ & $0351 \pm 0.136$ & $<$ & $0.476 \pm 0.113$ & $<$ & $0.501 \pm 0.127$ & $<$ & $0.539 \pm 0.133$ & $=$ & $0.517 \pm 0.149$ & $<$ & $0.502 \pm 0.132$ & $<$ & $0580 \pm 0.146$ & 12 \\
\hline & $\mathrm{Cl}_{95} \%$ & $\Uparrow$ & $0.702 \pm 0.04$ & $<$ & $0.704 \pm 0.032$ & $<$ & $0.725 \pm 0.048$ & $=$ & $0.775 \pm 0.033$ & $=$ & $0.776 \pm 0.033$ & $=$ & $0.779 \pm 0.032$ & $=$ & $0.782 \pm 0.037$ & $=$ & $0.779 \pm 0.036$ & $=$ & $0.787 \pm 0.037$ & 13 \\
\hline Spinal cord & & 50 & $35.9 \pm 4.5$ & $=$ & $35.4 \pm 4.0$ & $=$ & $35.1 \pm 3.9$ & $=$ & $36.5 \pm 4.9$ & $=$ & $35.5 \pm 4.0$ & $=$ & $35.6 \pm 4.1$ & $=$ & $36.0 \pm 4.1$ & $=$ & $35.7 \pm 4.5$ & $=$ & $35.5 \pm 3.9$ & 14 \\
\hline Brainstem & & 52 & $40.1 \pm 2.1$ & $=$ & $39.7 \pm 2.1$ & $=$ & $39.8 \pm 2.4$ & $=$ & $39.0 \pm 2.9$ & $=$ & $39.6 \pm 2.4$ & $=$ & $40.5 \pm 2.4$ & $=$ & $40.0 \pm 3.0$ & $=$ & $39.1 \pm 2.8$ & $=$ & $39.7 \pm 2.7$ & 15 \\
\hline $\begin{array}{l}\text { Plexus } \\
\text { ipsilateral }\end{array}$ & $D_{\max }$ & $61.6 \Downarrow$ & $58.5 \pm 5.0$ & $=$ & $58.6 \pm 5.6$ & $=$ & $58.6 \pm 5.6$ & $=$ & $59.0 \pm 5.1$ & $=$ & $59.0 \pm 5.2$ & $=$ & $59.0 \pm 5.1$ & $=$ & $59.0 \pm 5.1$ & $=$ & $59.0 \pm 5.2$ & $=$ & $58.9 \pm 5.1$ & 16 \\
\hline $\begin{array}{l}\text { Plexus } \\
\text { contralateral }\end{array}$ & & $50.4 \Downarrow$ & $51.3 \pm 1.1$ & $=$ & $51.0 \pm 0.7$ & $=$ & $50.8 \pm 1.4$ & $=$ & $50.9 \pm 0.5$ & $=$ & $51.1 \pm 0.5$ & $=$ & $51.2 \pm 0.7$ & $=$ & $51.5 \pm 1.0$ & $=$ & $51.5 \pm 0.9$ & $=$ & $51.3 \pm 0.8$ & 17 \\
\hline Mandibula & & $61.6 \Downarrow$ & $60.3 \pm 3.5$ & $=$ & $60.5 \pm 3.6$ & $=$ & $60.4 \pm 2.6$ & $=$ & $60.8 \pm 2.3$ & $=$ & $60.1 \pm 1.9$ & $=$ & $61.1 \pm 1.9$ & $=$ & $61.4 \pm 2.3$ & $=$ & $61.1 \pm 2.7$ & $=$ & $61.6 \pm 2.4$ & 18 \\
\hline \multirow{2}{*}{$\begin{array}{l}\text { Parotid } \\
\text { contralateral }\end{array}$} & $D_{\text {mean }}$ & 26 & $23.9 \pm 1.7$ & $<$ & $23.6 \pm 2.3$ & $<$ & $23.8 \pm 0.8$ & $<$ & $25.3 \pm 2.2$ & $=$ & $25.6 \pm 1.7$ & $=$ & $25.1 \pm 1.6$ & $=$ & $24.8 \pm 1.9$ & $=$ & $24.8 \pm 1.8$ & $=$ & $25.1 \pm 1.7$ & 19 \\
\hline & $V_{30}$ Gy & 50 & $34.2 \pm 6.3$ & $=$ & $33.6 \pm 7.4$ & $=$ & $33.9 \pm 4.5$ & $=$ & $36.3 \pm 6.4$ & $=$ & $37.9 \pm 5.8$ & + & $36.7 \pm 5.4$ & + & $33.8 \pm 6.8$ & $=$ & $34.3 \pm 6.0$ & $=$ & $34.9 \pm 6.0$ & 20 \\
\hline \multirow[t]{3}{*}{ Larynx } & $D_{\text {mean }}$ & $\Downarrow$ & $46.7 \pm 1.2$ & $<$ & $46.3 \pm 1.5$ & $<$ & $47.2 \pm 1.0$ & $=$ & $47.2 \pm 1.1$ & $=$ & $47.3 \pm 0.9$ & $=$ & $47.7 \pm 0.9$ & $=$ & $47.4 \pm 1.8$ & $=$ & $47.2 \pm 1.6$ & $=$ & $47.8 \pm 1.5$ & 21 \\
\hline & $V_{50 \text { Gy }}$ & $\Downarrow$ & $14.0 \pm 12.3$ & $=$ & $13.9 \pm 12.2$ & $=$ & $14.9 \pm 15.2$ & $=$ & $16.7 \pm 13.5$ & $=$ & $17.7 \pm 14.4$ & $=$ & $19.3 \pm 14.6$ & $=$ & $18.4 \pm 15.4$ & $=$ & $17.5 \pm 18.0$ & $=$ & $21.0 \pm 18.9$ & 22 \\
\hline & & & A & & B & & c & & D & & E & & $\mathrm{F}$ & & G & & $\mathrm{H}$ & & । & \\
\hline
\end{tabular}

Arrows indicate if a higher or a lower value gives the better plan. " $<$ ", $++^{\prime \prime}$ or " $=$ " indicate whether the result is lower, higher or equal to the VMAT ${ }^{A g i t t y}$ according to comparison a (bold) result on a significance level of $5 \%$, ${ }^{\text {a }}$ for segments: results are compared within each technique to the respective Agility plan according to comparison $Y$ (bold) 
Table 5 Results for nine plan groups (3 techniques $+3 \mathrm{MLC}$ ) for the evaluated parameters for the meningioma cases (average and standard deviation for five patients): QUANTEC criteria where applicable

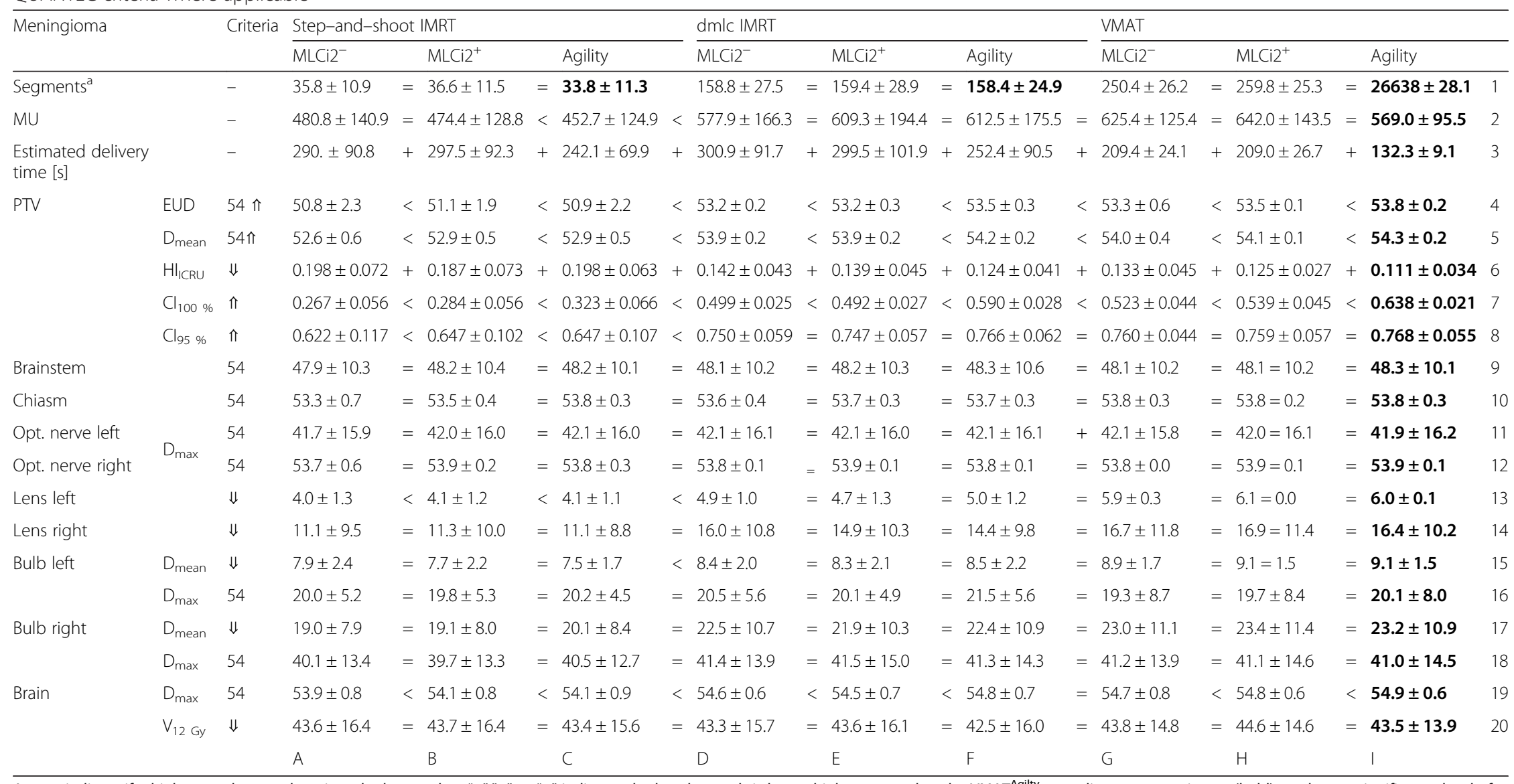

Arrows indicate if a higher or a lower value gives the better plan. " $\langle$ "," + " or " $="$ indicate whether the result is lower, higher or equal to the VMAT Agity according to comparison a (bold) result on a significance level of $5 \%,{ }^{a}$ for segments: results are compared within each technique to the respective Agility plan according to comparison $Y$ (bold) 
y) In order to distinguish differences caused by MLC independently of which technique was used, each MLC was compared to the corresponding Agility plan using the same technique. Therefore three underlying comparisons were made, which evaluate the influence of using $\mathrm{MLCi} 2^{-}, \mathrm{MLCi}^{+}$or Agility together with sIMRT, dMLC and VMAT. In the results Tables 4 and 5, comparison of MLCi2 $2^{-/+}$to Agility for ssIMRT would correspond to comparing column A/B vs. C $\left(\gamma^{\text {ssIMRT }}\right)$, while comparing MLCi2 $2^{-/+}$to Agility for dMLC or VMAT corresponds to D/E vs. F $\left(\gamma^{\mathrm{dMLC}}\right)$ and $\mathrm{G} / \mathrm{H}$ vs. I ( $\left.\gamma^{\mathrm{VMAT}}\right)$, respectively.

For each of the five patients per group, nine plans combining three different IMRT-techniques (ssIMRT, dMLC, VMAT) and three different MLCs (MLCi2 -/+ interdigitation, Agility-MLC) are generated. Thereby, plans are distinguished not only by MLC or technique but both - MLC and technique.

To conclude the results of the presented study, the mean and standard deviation for each evaluated parameter over the 5 patients per group as well as the results of the paired $T$-test (significance level: 0.05 ) for comparisons $\alpha, \beta$ and $\gamma$ are calculated. For a general overview, Tables 4 and 5 show the results for comparison $\alpha$ (comparison $\gamma$ for segments).

\section{Results}

In this study, 10 patients (five $\mathrm{HN}$, five MG) are optimized for ssIMRT (columns A-C, Tables 4 and 5), dMLC (columns D-F, Tables 4 and 5) and VMAT (columns G-I, Tables 4 and 5). For each technique, the MLCi2-MLC without (-) (columns A/D/G, Tables 4 and 5) and with (+) (columns $\mathrm{B} / \mathrm{E} / \mathrm{H}$, Tables 4 and 5 ) interdigitation and the Agility-MLC (columns C/F/I, Tables 4 and 5) are used. Thereby, 9 plans per patient are generated. The VMAT ${ }^{\text {Agility }}$ plan is considered as a reference for comparisons $\alpha$, as this is the plan used to determine all optimization parameters. An overview of all results of the head-and-neck and meningioma cases is shown in Tables 4 and 5, respectively. These tables include the results of a paired $T$-test, but only for comparison $\alpha$ with the VMAT ${ }^{\text {Agility }}$ plans. If the plan compared to is another one, results of the paired $T$-test are found in the text. For better understanding, indices from Tables 4 and 5 are used anyway.

As constrained optimization is used, dose to the OAR differed only slightly and all QUANTEC criteria are met. If OAR have to be spared more, the cost would be less PTV coverage. Among all plans, VMAT ${ }^{\text {Agility }}$ show best coverage and fastest delivery. Comparing different techniques $(\beta)$, VMAT always shows the fastest delivery. Comparing MLCs $(\gamma)$, Agility always shows the fastest delivery. Least MU are found for ssIMRT plans using the Agility-MLC. The total amount of MU depends on the PTV size and complexity as well as on the used technique. The total number of segments is independent of the used MLC for MG cases as well as dMLC and VMAT for HN cases.

Detailed results are as follows:

Head-and-Neck - OAR exposure

As constrained optimization is used, all OARs of the respective case fulfill the prescribed dose-limiting constraints. These constraints are optimized case-specific for this study, based on the VMAT ${ }^{\text {Agility }}$ plan (I1-I22, Table 4), and are not the ones clinically chosen, QUANTEC criteria are met for all OAR except for the mean dose of the larynx (A-I 21, Table 4).

This is because the PTV $_{50.4 \text { Gy }}$ surrounds the larynx in all $\mathrm{HN}$ cases, an example case (HN3) is shown in Fig. 1. Thereby, the QUANTEC criteria of $D_{\text {mean }}<44$ Gy (A-I 21, Table 4) is barely achievable, if the PTV is expected to receive the full dose. Due to the irradiation from all gantry angles, $\mathrm{D}_{\text {mean }}$ (A-I 21, Table 4) and $\mathrm{V}_{50 \text { Gy }}$ (A-I 22, Table 4) for the larynx are always highest for VMAT, even though significant differences $(\mathrm{p} \leq 0.01)$ are only found for ssIMRT $^{\text {MLCi2-/+ }}$ (D mean, A + B 21, Table 4).

For nerve structures (spinal cord - A-I 14, Table 4, brainstem - A-I 15, Table 4, plexus - A-I $16+17$, Table 4) differences in $D_{\text {max }}$ between techniques and MLCs are not statistically different from the VMAT ${ }^{\text {Agility }}$ plan according to the paired $T$-test and not larger than 1.5Gy. Within ssIMRT $\left(\gamma^{\text {ssIMRT }}, A-C\right.$, Table 4), Agility plans (C 14-17, Table 4) have equal or lower $\mathrm{D}_{\max }$. Also $\mathrm{D}_{\max }$ (mandibula) ( $\alpha$, A-I 18, Table 4) overall does not deviate more than $1.5 \mathrm{~Gy}$, but with lower doses in general for all ssIMRT plans $\left(\Delta \mathrm{D}_{\max }>1.0 \mathrm{~Gy}, \mathrm{~A}-\mathrm{C} 18\right.$, Table 4$)$.

The contralateral parotids (A-I $19+20$, Table 4) have the lowest $\mathrm{D}_{\text {mean }}$ in ssIMRT $\left(\Delta \mathrm{D}_{\text {mean }}>1.2 \mathrm{~Gy}, \mathrm{p} \leq 0.02\right.$, A-C 19 , Table 4). Evaluating $\mathrm{V}_{30 \mathrm{~Gy}}, \mathrm{dMLC}^{\mathrm{MLCi} 2+}$ and dmlc ${ }^{\text {Agility }}$ plans show $\mathrm{V}_{30 \text { Gy }}$ elevated at least by $5 \%(\mathrm{E}+\mathrm{F} 20$, $\mathrm{p}<0.01$, Table 4). Comparing MLCs within each technique $(\gamma)$, significant $(\mathrm{p} \leq 0.02$, Table 4$)$ differences are only found for the comparison $\mathrm{dmlc}^{\mathrm{MLCi}+}$ vs. dMLC ${ }^{\text {Agility }}(\mathrm{E} 19+20$ vs. F $19+20$, Table 4 ), showing higher $\mathrm{D}_{\text {mean }}$ and $\mathrm{V}_{30 \text { Gy }}$ for $\mathrm{dmlc}^{\mathrm{MLCi} 2+}$.

\section{Head-and-Neck - PTV coverage}

All OAR sparing compared to VMAT Agility comes at the cost of less PTV coverage. For all criteria (EUD, $\mathrm{D}_{\text {mean }}, \mathrm{HI}, \mathrm{CI}_{100} \%, \mathrm{CI}_{95}$ \% A 4-I 13, Table 4), VMAT ${ }^{\text {Agility }}$ (I 4-13, Table 4) has the best PTV coverage.

For PTV 50.4 Gy (A 4-I 8, Table 4), dMLC plans have at least 1.0Gy $(\mathrm{p} \leq 0.01)$ and $0.4 \mathrm{~Gy}(\mathrm{p} \leq 0.01)$ less EUD (D-F 4, Table 4) and $\mathrm{D}_{\text {mean }}(\mathrm{D}-\mathrm{F} 5$, Table 4), respectively, and ssIMRT plans have at least 2.1Gy $(\mathrm{p} \leq 0.01)$ and 1.2Gy $(\mathrm{p} \leq 0.01)$ less EUD (A-C 4, Table 4) and $\mathrm{D}_{\text {mean }}$ (A-C 5, Table 4), respectively. For $\mathrm{CI}_{95}$ \% $\left(\mathrm{PTV}_{50.4 \mathrm{~Gy}}\right)$, 


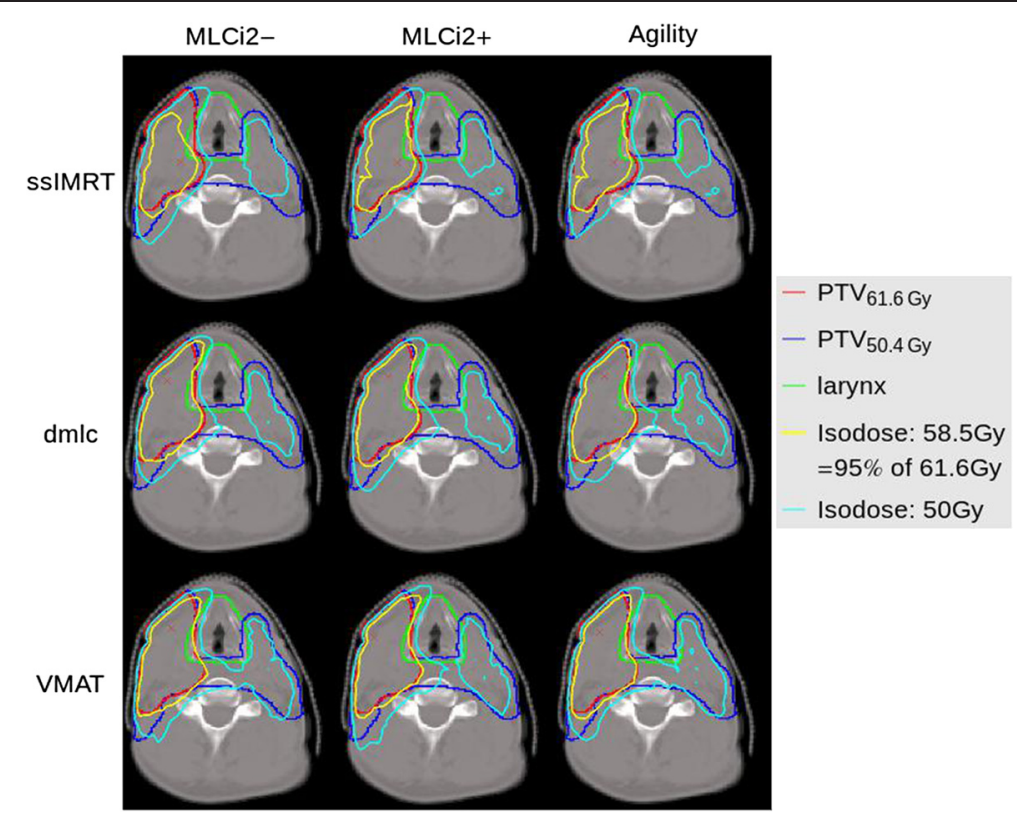

Fig. 1 By example of patient case HN3, relevant isodoses for the larynx (50Gy, 58.5Gy) are shown for the nine generated plans using ssIMRT, dMLC and VMAT with MLCi2 without (-) and with (+) interdigitation and the Agility-MLC

all dmlc plan show no significant difference compared to the VMAT Agility plan (D-E 8 vs. I 8, Table 4). Evaluating the coverage of PTV $61.6 \mathrm{~Gy}$, the $\mathrm{dMLC}^{\text {Agility }}$ plans show no significant differences in the evaluated parameters (F 9-13 vs. I 9-13, Table 4). For PTV $61.6 \mathrm{~Gy}$ only ssIMRT $^{\text {MLCi2-I+ }}$ have significant less conformity of the $95 \%$-isodose (A + B 13 vs. I 13, Table 4).

Comparing different MLCs within the techniques $(\gamma$ : $\mathrm{A}+\mathrm{B} v s . \mathrm{C}, \mathrm{D}+\mathrm{E} v s . \mathrm{F}, \mathrm{G}+\mathrm{H}$ vs. I, Table 4), significant differences for the PTV coverage in ssIMRT $\left(\gamma^{\text {ssIMRT }}\right.$ : $\mathrm{A}+\mathrm{B}$ 4-13 vs. C 4-13, Table 4) are found for $\mathrm{CI}_{95} \%$ $\left(\mathrm{PTV}_{50.4 \mathrm{~Gy}}\right) \quad(\mathrm{p} \leq 0.01) \quad(\mathrm{A} 8$ vs. C 8 , Table 4$)$ and $\operatorname{EUD}\left(\mathrm{PTV}_{61.6 \mathrm{~Gy}}\right)(\mathrm{p} \leq 0.01)$ (A 9 vs. C 9, Table 4). Within the $\mathrm{dMLC}$ plans $\left(\gamma^{\mathrm{dMLC}}\right), \mathrm{MLCi2}^{+}$shows significant differences only for $\mathrm{PTV}_{61.6 \mathrm{~Gy}}\left(\mathrm{D}_{\text {mean }}, \mathrm{HI}\right.$ and $\mathrm{CI}_{100} \%$, E 10-12 vs. F 10-12, Table 4), while MLCi2 ${ }^{-}$has less PTV coverage $(\mathrm{p}=0.01)$ in terms of $\mathrm{D}_{\text {mean }}(-1.2 \mathrm{~Gy})$ and $\mathrm{CI}_{100} \%$

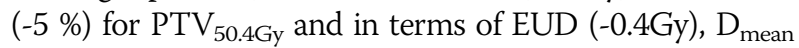
(-0.2Gy), HI (-10\%) and $\mathrm{CI}_{100} \%$ (-12 \%) for PTV $\mathrm{Pl}_{6 \mathrm{GGy}}(\mathrm{D}$ 4-13 vs. F 4-13, Table 4).

Comparing different techniques using the same MLC $(B: A+D$ vs. $G, B+E$ vs. $\mathrm{H}, \mathrm{C}+\mathrm{F}$ vs. I, Table 4), ssIMRT plans have worse PTV coverage $(\mathrm{p} \leq 0.01)$ using either of the three MLCs. Using MLCi2 ${ }^{-}\left(\beta^{\mathrm{MLCi2}-}\right)$, dMLC plans show less homogeneity $(\mathrm{p} \leq 0.02)$ for $\mathrm{PTV}_{50.4 \mathrm{~Gy}}$ and PTV $_{\text {61.6Gy }}$ and less EUD (-0.4Gy) for PTV61.6Gy compared to $\mathrm{VMAT}^{\mathrm{MLCi} 2}$. For $\mathrm{dMLC} \mathrm{CLCi}^{\mathrm{ML}+}\left(\beta^{\mathrm{MLCi} 2+}\right)$, significant $(\mathrm{p} \leq 0.01)$ differences for the PTV coverage compared to $\mathrm{VMAT}^{\mathrm{MLCi} 2+}$ are only found for $\mathrm{HI}\left(\mathrm{PTV}_{50.4 \mathrm{~Gy}}\right)$ (-4 \%) (E 4-13 vs. H 4-13, Table 4), whereas the PTV coverage for dMLC using the Agility-
MLC $\left(\beta^{\text {Agility }}\right)$ is less in terms of EUD, $D_{\text {mean }}, \mathrm{HI}$ and $\mathrm{CI}_{100} \%$ for both PTVs compared to VMAT ${ }^{\text {Agility }}$ (F 4-13 vs. I 4-13, Table 4).

Figure 2 shows the results for one HN patient (HN3 in Tables 3) as an example. Figure $2 d$ depicts that the evaluated OAR parameters differ only slightly between the techniques and used MLC. As mentioned, larger differences are found for $\mathrm{V}_{50 G y}$ (larynx) (A-I 22, Table 4), $\mathrm{D}_{\max }$ (mandibula) (A-I 18, Table 4) and the contralateral parotid ( $\left.D_{\text {mean }}, V_{30 G y}\right)$ (A-I $19+20$, Table 4). In contrast to the only slightly differing clinically important OAR parameters, Fig. 2a, b and c depict the differences of the PTV coverage: $\mathrm{HI}, \mathrm{CI}$ and $D_{\text {mean }}$ drop clearly, if ssIMRT is used. Influences of the MLC design are only small and highest in combination with VMAT.

\section{Meningioma - OAR exposure}

As for the $\mathrm{HN}$ cases, all generated plans fulfill the prescribed constraints and predefined criteria. Larger standard deviations for the brainstem (A-I 9, Table 5), left optical nerve (A-I 11, Table 5), right lens (A-I 14, Table 5) and bulb (A-I $17+18$, Table 5) are found due different proximity of the PTV to these OARs for the different patients $(\alpha)$.

Differences between the techniques and MLCs are found for the eyes (A-I 13-18, Table 5). Among all plans $(\alpha)$, ssIMRT plans preserve lenses and bulbs best $\left(\Delta \mathrm{D}_{\text {mean }}\right.$ (bulbs) $\leq 4.2 \mathrm{~Gy}, \mathrm{p} \geq 0.05$ (A-C $15 / 17$, Table 5), $\Delta \mathrm{D}_{\max }($ lenses $) \leq 5.3 \mathrm{~Gy}, \mathrm{p} \leq 0.02(\mathrm{~A}-\mathrm{C} 13+14$, Table 5$)$ ), 
a) DVH: PTV $61.6 G y$ and PTV $50.46 y$

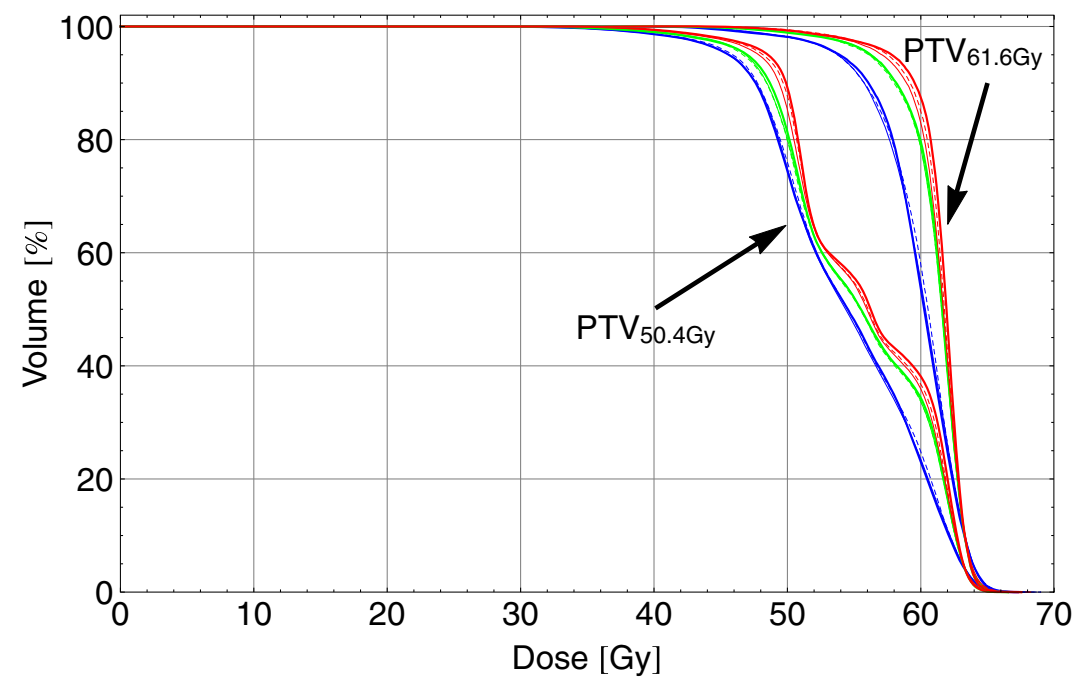

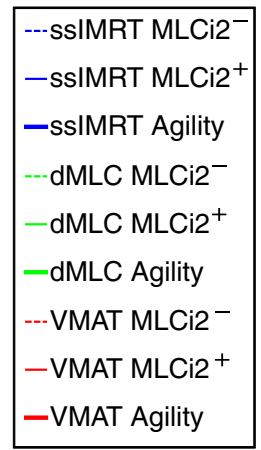

b) PTV coverage: PTV $61.6 G y$

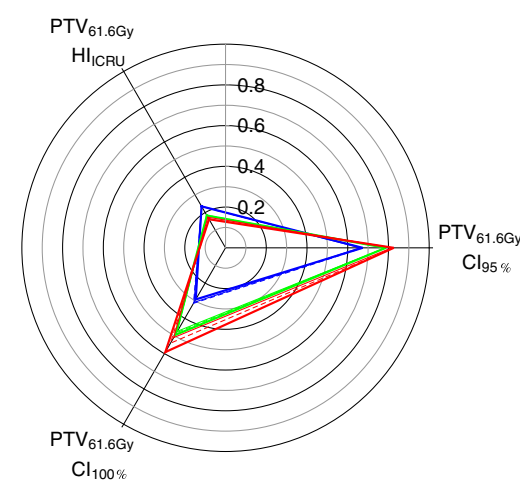

c) PTV coverage: PTV 50.4 Gy

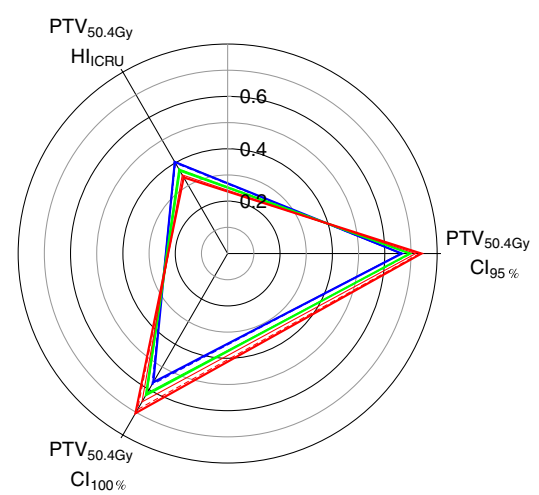

d) OAR exposure

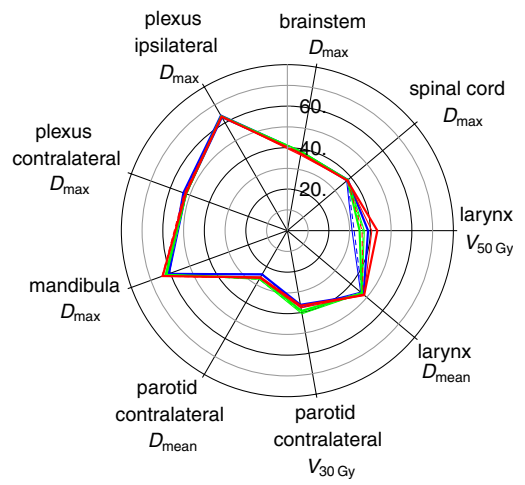

Fig. 2 Example of one head-and-neck case (HN3). a DVHs for PTV 61.66 and PTV $50.46 y$ of all nine generated plans, (b) homogeneity and conformity indices for PTV61.6Gy and c) PTV 50.4Gy, d) evaluated QUANTEC and clinical criteria for OARs

while $\mathrm{dMLC}$ plans preserve the lenses $\left(\Delta \mathrm{D}_{\max }=0.4\right.$ 2.0Gy, $\mathrm{p} \geq 0.07$ (D-F $13+14$, Table 5)) and the bulbs $\left(\Delta \mathrm{D}_{\text {mean }}=0.6-1.4 \mathrm{~Gy}, \mathrm{p} \geq 0.06(\mathrm{D}-\mathrm{F} 15 / 17\right.$, Table 5) $)$ better than VMAT. Reasons are (1) the use of the advanced segment shape optimization algorithm for VMAT during the second optimization step that enables to fully take advantage of the constraints by placing the leaves more effectively with respect to PTV coverage and therefore nearer to the OAR, and (2) VMAT uses the full range of possible gantry angles, while the gantry angles for ssIMRT and AMLC were chosen, such that they would avoid radiation to the eyes more effectively. The use of Agility shows an influence when using $\mathrm{dMLC}\left(\gamma^{\mathrm{dMLC}}, \mathrm{F} v s\right.$. $\mathrm{D}+\mathrm{E}$, Table 5), presumably due to its interdigitation capabilities and smaller leaves. Overall, only the differences for the left lens for the ssIMRT plans are significant when comparing to $\mathrm{VMAT}^{\text {Agility. }}$
Furthermore, significantly $(\mathrm{p} \leq 0.01)$ lower $\mathrm{D}_{\max }$ (upto 1Gy) are found for brain (A-I 20, Table 5). Only for $\mathrm{dMLC}^{\text {Agility }}$ and VMAT ${ }^{\mathrm{MLCi2}-}$, these changes are significant $(\mathrm{p} \leq 0.05)$. The maximal dose of the brain lies within the PTV. As the PTV coverage is best for VMAT ${ }^{\text {Agility }}$ (see next paragraph), higher $D_{\text {max }}$ (brain) of these plans can be explained by this.

\section{Meningioma - PTV coverage}

As for the $\mathrm{HN}$ cases, PTV coverage for the meningioma cases is best for VMAT ${ }^{\text {Agility }}$ (A-I 4-8, Table 5). EUD, $\mathrm{D}_{\text {mean }}$, $\mathrm{HI}$ and $\mathrm{CI}_{100}$ \% show less PTV coverage for all other plans. Differences for $\mathrm{CI}_{95} \%$ are non-significant for dMLC and VMAT using either MLC (D-I 8, Table 5). Larger differences for the PTV coverage $(\triangle \mathrm{EUD} \geq 2.7 \mathrm{~Gy}$ $\left.(\mathrm{p} \leq 0.02), \Delta \mathrm{D}_{\text {mean }} \geq 1.4 \mathrm{~Gy}(\mathrm{p} \leq 0.02)\right)$ are found for all 
ssIMRT plans (A-C 4-8 vs. I 4-8), showing that also for meningioma, the technique is more essential than the MLC design. For all ssIMRT, evaluated parameters show much less PTV coverage than dMLC or VMAT (A-C 4-8 vs. D-I 4-8, Table 5).

Comparing MLCs within the used techniques $(\gamma: A+B$ vs. C, D+E vs. F, G+H vs. I, Table 5), the Agility-MLC shows significantly higher PTV coverage for the conformity $(\mathrm{p} \leq 0.02)$ of ssIMRT $\left(\gamma^{\text {ssIMRT }}\right)$, but also for EUD, $D_{\text {mean }}$, HI und $\mathrm{CI}_{100} \%$ within dMLC $\left(\gamma^{\mathrm{dMLC}}\right)$ or VMAT plans $\left(\gamma^{\mathrm{VMAT}}\right)(\mathrm{p} \leq 0.01$ (dMLC), $\mathrm{p}=0.02$ (VMAT)). Therefore, the use of the Agility-MLC has more impact for dMLC and VMAT.

Comparing different techniques using the same MLC ( $B: A+D$ vs. $G, B+E$ vs. $\mathrm{H}, \mathrm{C}+\mathrm{F}$ vs. I, Table 5), MLCi2 has $(\mathrm{p} \leq 0.01)$ less PTV coverage for ssIMRT $\left(\beta^{\mathrm{MLCi2}-}\right)$. Using the MLCi2 with interdigitation, differences within dMLC plans become significant $(\mathrm{p} \leq 0.02)$ for EUD, $\mathrm{D}_{\text {mean }}$ and $\mathrm{CI}_{100} \%\left(\beta^{\mathrm{MLCi} 2+}\right)$.

Even though not significant $(\mathrm{p} \geq 0.07)$, dMLC ${ }^{\text {Agility }}$ show better homogeneity and conformity than any $\mathrm{VMAT}^{\mathrm{MLCi} 2}$ (F 6-8 vs. H 6-8, Table 5), showing that (1) the used gantry angles are chosen such, that comparable plans to VMAT $^{\text {Agility }}$ are possible and (2) smaller leaves are favorable when small OAR within the PTV need to be preserved. Still the PTV coverage in terms of EUD and $D_{\text {mean }}$ for dMLC (all MLCs) are within the range of the VMAT plans $(\triangle \mathrm{EUD}=0.2-0.6 \mathrm{~Gy}$ (D-F 4 vs. G-I 4 , Table 5), $\Delta \mathrm{D}_{\text {mean }}=0.2-0.4$ Gy (D-F 5 vs. G-I 5, Table 5)). For all ssIMRT, evaluated parameters show much less PTV coverage than dMLC or VMAT (A-C 4-8 vs. D-I 4-8, Table 5).

Figure 3 shows the results for one meningioma patient (MG2 in Tables 2) as an example. Firgure 3c depicts that the evaluated OAR parameter differ only for the bulbs $\left(D_{\text {mean }}, D_{\max }\right)$ and the lenses $\left(D_{\max }\right)$. In contrast, Fig. 3a and $b$ depict the differences of the PTV coverage: homogeneity, conformity and $\mathrm{D}_{\text {mean }}$ drop clearly, if ssIMRT is used. Influences of the MLC design are only small and highest in combination with VMAT.

\section{Estimated treatment times}

The reported treatment times are not measured, but estimated from the TPS with realistic assumptions for the dose rate, leaf and gantry rotation velocity. Hence, times within this study are at least comparable among each other. As absolute times depend on the maximal achievable dose rate, leaf and gantry rotation velocity of a specific linac, the actual delivery times may deviate about few percent from those treatment times estimates. However, differences between techniques or MLCs from the presented study may be compared to other studies in a relative manner.
The analysis of the estimated treatment time shows, that the combination of VMAT and Agility results in the fastest treatment for each case. This is still true when using up to three arcs for $\mathrm{HN}$ cases and the time difference can be up to $481 \mathrm{~s}(\approx 8 \mathrm{~min})$ for single cases (HN2), comparing VMAT ${ }^{\text {Agility }}$ vs. ssIMRT ${ }^{\text {MLCi2- }}$.

Comparing each technique using either MLC ( $\mathrm{B}$ : $\mathrm{A}+\mathrm{D} 3$ vs. G 3, B + E 3 vs. H 3, C + F 3 vs. I 3, Tables 4 and 5), fastest $(p \leq 0.01)$ treatment plans are found among VMAT, regardless of the PTV complexity (shape, dose levels, proximity to OARs), while ssIMRT takes the most time (A-C 3 vs. D-F 3 vs. G-I 3, Tables 4 and 5). The MLC influences the amount of time that can be reduced by changing from one technique to another. $\mathrm{VMAT}^{\mathrm{MLCi2}-\mathrm{I}_{+}}$ reduces the treatment time about $60 \%$ compared to ssIMRT with $\mathrm{MLCi} 2^{-1+}\left(\mathrm{B}^{\mathrm{MLCi2}-{ }^{+}+}: \mathrm{A}+\mathrm{B} 3\right.$ vs. G + H 3, Tables 4 and 5 ) and almost $50 \%$ with Agility ( $\beta^{\text {Agility }}$ : C 3 vs. I 3, Tables 4 and 5). For dMLC, treatment times are reduced by about $15 \%\left(\beta^{\mathrm{MLCi2}-/+}: \mathrm{D}+\mathrm{E} 3\right.$ vs. $\mathrm{G}+\mathrm{H} 3$, Tables 4 and 5 ) and $45 \%$ ( $\beta^{\text {Agility }}$ : F 3 vs. I 3, Tables 4 and 5) using MLCi2 ${ }^{-1+}$ and Agility, respectively.

Comparing the MLCs using either technique $(\gamma)$, Agility on average offers faster $(\mathrm{p} \leq 0.01)$ treatments by $48 / 55 \mathrm{~s}$ for ssIMRT ( $\gamma^{\text {ssIMRT }}$ : A + B 3 vs. C 3, Table 5), 48/47 s for $\mathrm{dMLC}\left(\gamma^{\mathrm{dMLC}}: \mathrm{D}+\mathrm{E} 3\right.$ vs. F 3, Table 5) and 77/76 s for VMAT ( $\gamma^{\mathrm{VMAT}}: \mathrm{G}+\mathrm{H} 3$ vs. I 3, Table 5) for meningioma using the $\mathrm{MLCi}^{-1+}$. This corresponds to about $20 \%$ longer treatments for ssIMRT and dMLC and $58 \%$ longer treatments for VMAT with MLCi2. For the larger and more complex PTVs of the HN cases, Agility is faster by $160 / 124 \mathrm{~s}$ for $\operatorname{ssIMRT}\left(\gamma^{\text {sIMRT }}\right.$ : A + B 3 vs. C 3, Table 4), 98/97 s for dMLC ( $\gamma^{\mathrm{dMLC}}: \mathrm{D}+\mathrm{E} 3$ vs. F 3, Table 4) and 143/137 s for VMAT ( $\gamma^{\mathrm{VMAT}}: \mathrm{G}+\mathrm{H} 3$ vs. I 3, Table 4) for MLCi2 and $\mathrm{MLCi}^{+}$, respectively, corresponding to 28 and $36 \%$ longer treatments for ssIMRT, $30 \%$ longer treatments for dMLC and over $60 \%$ longer treatments for VMAT when using the MLCi2.

The ability of interdigitation of the MLCi2 does not reduce treatment times much. Larger time reduction is found for ssIMRT, only (HN: $36 \mathrm{~s}, 10 \%, \mathrm{p}=0.07$, A 3 vs. B 3, Table 4, MG: 7 s, $3 \%, \mathrm{p} \leq 0.01$, A 3 vs. B 3, Table 5).

\section{Monitor units}

MU for HN and MG are least for ssIMRT ${ }^{\mathrm{MLCi2}+}$ and ssIMRT $^{\text {Agility }}$ (B/C 2, Tables 4 and 5).

Compared to MLCi2 ${ }^{-}$and MLCi2 ${ }^{+}$, Agility saves MU for HN cases using ssIMRT ( $32 \%, 13 \%, \mathrm{p} \leq 0.01\left(\gamma^{\text {ssIMRT }}\right.$ : A/ B 2 vs. C 2, Table 4)) and VMAT (7\%, $10 \%, \mathrm{p} \leq 0.01$ $\left(\gamma^{\mathrm{VMAT}}\right.$ : G/H 2 vs. I 2, Table 4)), but not for $\operatorname{dMLC}\left(\gamma^{\mathrm{dMLC}}\right.$ : D/E 2 vs. F 2, Tables 4 and 5) or MG cases ( $\gamma:$ A/B 2 vs. C 2, D/E 2 vs. F 2, G/H 2 vs. I 2, Table 5). 


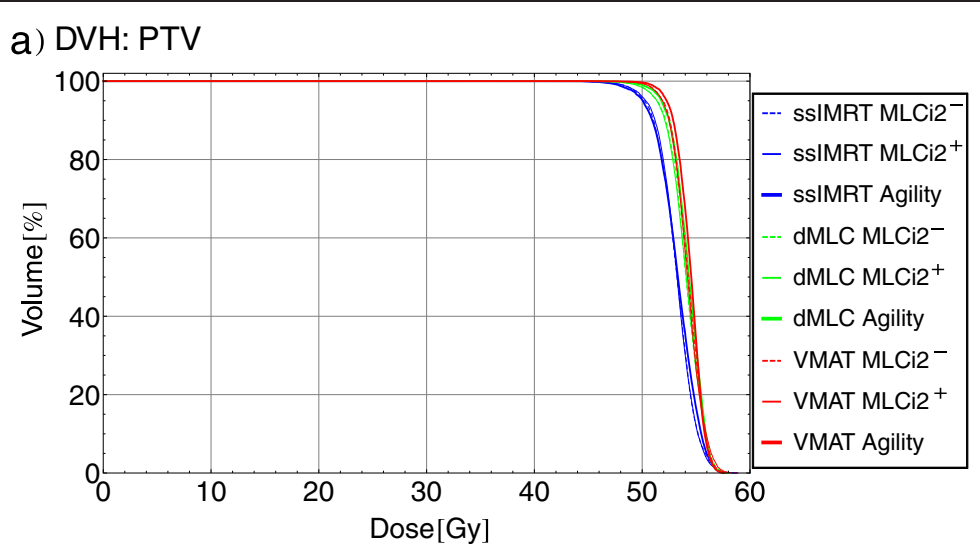

b) PTV coverage

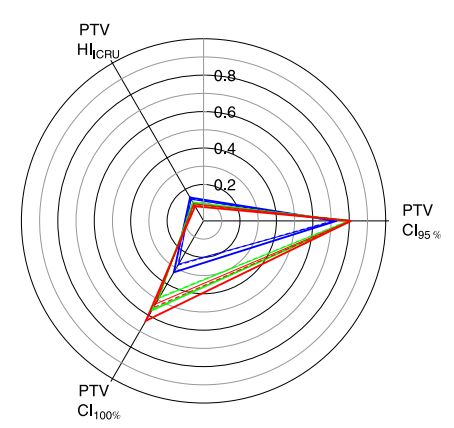

C) OAR exposure

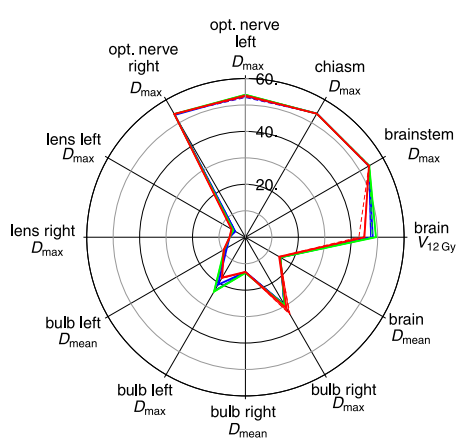

Fig. 3 Example of one meningioma case (MG2). a DVH for the PTV of all nine generated plans, (b) homogeneity and conformity indices for the PTV, c) evaluated QUANTEC and clinical criteria for OARs

Comparing each technique using either MLC for MG cases (ß), ssIMRT plans have the least MU as compared to the respective VMAT plans $(\triangle \mathrm{MU}=23 \% / 26 \% / 20 \%$, $\mathrm{p} \leq 0.01, \mathrm{MLCi}^{-} / \mathrm{MLCi}^{+} /$Agility, A/B/C 2 vs. G/H/I 2, Table 5), while no significant changes $(p \geq 0.36)$ between $\mathrm{dMLC}$ and VMAT are found. For HN cases VMAT increases $\mathrm{MU}$ compared to dMLC $(\triangle \mathrm{MU}=19 \% / 18 \% /$ $10 \%, \mathrm{p} \leq 0.01$, MLCi2/MLCi2 ${ }^{+} /$Agility, D/E/F 2 vs. G/H/ I 2, Table 4) and ssIMRT ( $\triangle M U=9 \% / 25 \% / 26 \%, \mathrm{p} \leq$ 0.01, MLCi2/MLCi2 ${ }^{+} /$Agility, A/B/C 2 vs. G/H/I 2, Table 4). Interdigitation reduces the $\mathrm{MU}$ for $\mathrm{HN}$ cases for ssIMRT by $15 \%(\mathrm{p} \leq 0.01)$ (A 2 vs. B 2, Table 4). This shows that the reduction of MU depends not only on the size and complexity of the treated volume but also on the technique and the MLC.

\section{Segments}

The number of segments for the different MLCs for a given treatment technique $(\gamma)$ is also investigated (A-I 1, Tables 4 and 5).

For dMLC and VMAT $\left(\gamma^{\mathrm{dMLC} / \mathrm{VMAT}}\right)$, the amount of segments does not change significantly with the MLC and only slightly with the plan complexity (mean number of segments: MG: 159 (dMLC, D-F 1, Table 5)/259 (VMAT, G-I 1, Table 5), HN: 170 (D-F 1, Table 4)/356 (VMAT, G-I 1, Table 4)).

For ssIMRT $\left(\gamma^{\text {sIMRT }}\right)$, segments do not change with the MLC (34-37 segments, $\mathrm{p} \geq 0.15$ (A-C 1, Table 5)) for the MG cases, but for the $\mathrm{HN}$ cases $(+19 / 8$ segments $\mathrm{MLCi}^{-1+}, \mathrm{p} \leq 0.01$,(A-C 1, Table 4)). Interdigitation reduces the amount of segments by $12 \%(\mathrm{p} \leq 0.01)$ for HN cases using ssIMRT (A 1 vs. B 1, Table 4).

\section{Discussion}

This work evaluates the impact of the MLC design (MLCi2 without (-) and with (+) interdigitation, and Agility-MLC) with regard to different IMRT techniques (ssIMRT, dMLC, VMAT) for head-and-neck and meningioma cases by means of PTV coverage, dose to OARs and some plan related parameters (MU, segments, treatment time).

One single person generated all plans using the same TPS version and dose algorithms, in order to keep the bias of this study low. Inevitably, differences are introduced by different optimization and sequencing 
algorithms needed for different techniques. Thus, these results are to some extent specific for the TPS (and its version) used in this study. By design of this study, the aim was to create plans where the dose to OARs within each case is comparable, thereby revealing the specific impact of MLC design and IMRT technique in terms of the target coverage. Thus, constrained optimization was used to find a Pareto optimal plan, which had all doselimiting constraints minimized individually on a casespecific basis, such that the PTV coverage was just not affected for the VMAT ${ }^{\text {Agility }}$ plan. As ssIMRT and dMLC use static gantry angles, the chosen angles may affect the results. However, as the results show, dMLC ${ }^{\text {Agility }}$ plans degrade only slightly compared to VMAT ${ }^{\mathrm{MLCi2-/+}}$, showing that the selected gantry angles were chosen reasonably with respect to PTV and OAR and therefore differences result from the used IMRT technique and MLC design.

As intended by constrained optimization, OAR exposure is within the prescriptions and therefore mainly the same for all 9 plans of each case (Figs. 2d and 3c). Dose differences within the prescribed limits are found, because the used prescription functions (modelling the biological effect of radiation to different tissues) do not match the clinical evaluated parameters. As the prescribing functions consider the organ exposure as a whole in terms of EUD (applying model specific parameters) and not in terms of single dose-volume-parameters, DVH curves may differ but result in the same EUD. Especially if critical structures are close to the PTV or belong partially to the PTV (larynx, parotid glands, mandibula, eyes), OAR exposure of a certain part of the DVH rises slightly with increased PTV coverage for the VMAT plans. However, as other parts of the same OAR can be preserved better by more complex techniques, the EUD remains the same. The higher OAR exposure for certain DVH parts found for more complex techniques is not only caused by higher scatter due to employing more beam directions and MU [31-33], but also due to the recently introduced advanced segment shape optimization that places leaves in VMAT optimization more effectively, such that constraints are not violated but PTV coverage is improved. By this, the distance between the projected leaf tip position and the OAR can be smaller for VMAT than for SsIMRT and dMLC.

The main results of this study show that (1) the smaller leaves of the Agility-MLC are capable of sparing the OARs to the same extent as with the MLCi2 ${ }^{+/-}$, while increasing the PTV coverage, (2) using Elekta-VMAT planned with the TPS Hyperion V2.4 (equivalent to using Monaco 3.2) does not suffer the loss of either OAR sparing or PTV coverage and (3) Elekta-VMAT using the Agility-MLC planned with the TPS Hyperion V2.4 offers faster treatments than using the MLCi2 ${ }^{+/-}$or ssIMRT and dMLC.
Some authors $[1,5,6,15,16,33-37]$ studied the impact of different MLC properties showing 4 or $5 \mathrm{~mm}$ leaf width to be slightly superior over $10 \mathrm{~mm}$ leaf width in terms of either better PTV coverage, homogeneity and conformity or OAR sparing. Especially small OAR or target structures with small concavities will profit. As the aim of the presented study was to isolate differences in PTV coverage, homogeneity and conformity, narrower leaves give plans with higher mean dose to the target as well as improved homogeneity and conformity, for most cases. Consistent to van Kesteren et al. [15] and Lafond et al. [16], interdigitation had only little impact on improved PTV coverage, but could improve delivery efficiency by means of reduced MU [16], because in critical situations, OAR sparing can be performed more precisely and efficiently, if leaves can move freely. If interdigitation of the leaves is possible, the algorithm can choose the most efficient mode of sparing.

Higher impact on improved PTV coverage is found by technique. Shown by others [31, 33, 35, 38-42], comparable or slightly improved PTV coverage with better conformity and homogeneity is found comparing static or dynamic IMRT techniques to rotational IMRT. The recently introduced advanced segment shape optimization routine further improves homogeneity, especially for meningioma cases. Here, leaves are more effectively placed for better OAR sparing and better PTV coverage. Consequently, best PTV coverage is obtained for VMAT $^{\text {Agility }}$ optimized with the advanced segment shape optimization routine. ${ }^{1}$

Forty to $50 \%$ treatment time reduction is reported using VMAT instead of ssIMRT and MLCi2 for cases with medium or high PTV complexity (prostate, HN, lung) $[31,33,38,39,42]$. This current study also shows 37 to $50 \%$ faster treatments for highly complex PTVs (HN) and 28 to $45 \%$ for less complex PTVs (MG) using VMAT instead of ssIMRT. Wizorek et al. [43] report over $75 \%$ faster treatments with RapidArc compared to dynamic IMRT for HN cases, whereas this study finds 13 and $30 \%$ (MLCi2 $^{-/+}$and Agility) for $\mathrm{HN}$ and 30 and $48 \%$ (MLCi2 $^{-/+}$and Agility) for MG. Reasons for this discrepancy of time speed up between the according Varian techniques (dynamic IMRT compared to Rapi$\mathrm{dArc}$ ) on the one hand, and the Elekta techniques (dMLC compared to VMAT) on the other hand, may be manifold. First, RapidArc uses a different approach, in which the gantry speed modulation is considered to a smaller degree as for VMAT, mainly trying to keep gantry rotation speed at maximum. Second, this study uses two to three arcs and nine gantry angles instead of always two arcs and seven fields as Wizorek et al. Third, constraints are tighter in this study, resulting in more complex and therefore longer treatments. Forth, treatment times for dynamic IMRT in head-and-neck IMRT as reported by 
Wizorek et al. are at a higher level $(10.5 \pm 1 \mathrm{~min})$ than treatment times calculated for this study using dMLC $(7.0 \pm 0.4 \mathrm{~min})$. Therefore, a relatively higher gain in treatment time is possible. These higher treatment times for dMLC-IMRT calculated with Eclipse TPS is also shown by Shang et al. [44] (rectal cancer: 8.0 \pm $0.7 \mathrm{~min}$ ) and Jeong et al. [45] (HN: 11.7 to $19.6 \mathrm{~min}$, depending on the number of fields).

The Agility-MLC accelerates treatments compared to $\mathrm{MLCi} 2^{-/+}$in this study by $22-26 \%(\mathrm{HN})$ and $17-19 \%$ (MG) for ssIMRT und dMLC, presumably due to higher leaf speed. Only evaluating VMAT, treatments are about $39 \%$ faster for $\mathrm{HN}$ and meningioma. Bedford et al. [27] report $53 \%$ accelerated VMAT treatments for typical HN. Tighter constraints in the presented study can cause longer treatment times for all techniques, and Bedford et al. use only one arc, a maximum delivery time constraint as well as a different optimization approach.

Even though reported treatment times are not measured but TPS-calculated and therefore only estimates, calculations were done with realistic assumptions for the dose rate, leaf and gantry rotation velocity. All these parameters were the same throughout the study. The absolute treatment times depend on the maximal achievable dose rate, leaf and gantry rotation velocity of a specific linac and may vary due to daily output variations, the actual wear of the single moving components as well as MLC calibration and beam tune. Hence, treatment times are comparable within this study and at least comparable in a relative manner to other studies.

A decrease in MU when treating $\mathrm{HN}$ with VMAT (1-2 arcs) instead of ssIMRT of 9 to $20 \%$ has been reported [31, 33, 42]. Contrary, we find increased MU (10\% $\mathrm{MLCi}^{-}$(p = 0.06), $25 \% \mathrm{MLCi}^{+}$(p $\left.\leq 0.01\right), 26 \%$ Agility $(\mathrm{p} \leq 0.01))$ as also found by Guckenberger et al. [31] (7 \% MLCi2 $\left.{ }^{-}\right)$when using three arcs for VMAT. Large MU reductions (35-60 \%) compared to dMLC are reported for RapidArc [40, 43-47]. In the presented study, again, increased MU (10-19\%) are found if using VMAT instead of dMLC for HN cases. For MG, no significant MU reduction between $\mathrm{AMLC}$ and VMAT is found. One reason is that $\mathrm{dMLC}$, calculated with Eclipse for Varian linacs, typically produces over $1100 \mathrm{MU}$ as compared to $800 \mathrm{MU}$ in this study, while for RapidArc less MU than for Elekta-VMAT are needed [40].

Burmeister et al. [1] found increased MU for narrower leaves while Wang et al. [36] report less MU for narrower leaves $(4 \mathrm{~mm})$. Also the presented data show improved MU efficiency with the narrower leaf MLC (Agility) for ssIMRT and VMAT. One reason could be that Burmeister normalized the plans. As in that study, homogeneity and $\mathrm{D}_{\text {min }}$ of the PTV was less for the narrower leaf plans, the normalization can involuntarily cause higher mean dose in the PTV and thereby higher MU. Besides, lower transmission leads to lower doses (esp. low dose region) in OARs [5] possibly enabling the constrained optimization algorithm to achieve higher PTV coverage for a given amount of extra dose due to MLC transmission. Therefore, presumably the lower transmission of the AgilityMLC compared to the MLCi2 is also one reason for improved MU efficiency.

\section{Conclusion}

Best plans in terms of PTV coverage (EUD, $\mathrm{D}_{\text {mean }}, \mathrm{HI}$, $\mathrm{CI}$ ) while maintaining the OAR exposure and treatment delivery time are found for VMAT plans, delivered with the Agility-MLC. Due to reduction in transmission and improvements in leaf speed, these plans have only 3 and $16 \%$ (HnN/meningioma) more MU than the corresponding ssIMRT, delivered with a non-interdigitating MLCi2.

\section{Endnotes}

${ }^{1}$ This advanced segment shape optimization routine is also available for ssIMRT in Monaco 5.1 and higher, and for dMLC in Monaco 5.0 and higher. With this, improved plans for ssIMRT and dMLC will be found.

\begin{abstract}
Abbreviations
$\mathrm{Cl}$ : Conformity index; $D_{\max }$ : Maximal dose (as dose to $99 \%$ of the volume); $D_{\text {mean }}$ : Mean dose; $D_{\min }$ : Minimal dose (as dose to $1 \%$ of the volume); dMLC: Dynamic sliding window IMRT; DVH: Dose volume histogram; EUD: Equivalent uniform dose; Fig: Figure; GTV: Gross tumor volume; Gy: Gray; HI: Homogeneity index; HN: Head and neck; ICRU: International Commission on Radiation Units and Measurements; IMRT: Intensity modulated radiotherapy; MG: Meningioma; MLC: Multileaf collimator; MR: Magnet resonance; MU: Monitor units; OAR: Organ at risk; PET: Positron emmision tomography; PTV: Planning target volume; QUANTEC: Quantitative analysis of normal tissue effects in the clinic; SIB: Simultan integrated boost; SsIMRT: Step-and-shoot IMRT; Tab: Table; TPS: Treatment planning system; VMAT: Volumetric modulated arc therapy; $V_{x G y}$ : Volume receiving a dose of $x G y$.
\end{abstract}

\section{Competing interests}

All authors declare that they have no competing interest.

\section{Authors' contribution}

The concept of this study was designed by MA, MS, UG and SK. Patient enrolment was done by CB, SK and UG. All IMRT and VMAT plans were generated by SK. Clinical plan revision was done by UG. Data collection and acquisition was done by SK, MS and UG. SK, MS, AT, UG, MR and HW were involved with data analysis. The manuscript was drafted by SK and revised by MS, AT, UG, MA and MR. Literature revision was done by SK, MS and UG. MR, HW, MA, AT and CB gave additional guidance and support. All authors read and finally approved the manuscript.

\section{Acknowledgements}

This work was in parts supported by Elekta, Germany.

\section{Disclosure}

Elekta Germany supports research at the Department of Radiation Oncology at the Medical University Center of the Ludwig-Maximilians-University, Munich. Elekta supported various congress presentations by MA, CB, UG, MR and SK. Other authors state no conflict of interest. Elekta had no influence on study design or collection, analysis or interpretation of data; nor did Elekta have any influence on the writing of the manuscript for publication. 


\section{Author details}

'Department of Radiation Oncology, Ludwig-Maximilians-University, Munich Germany. ${ }^{2}$ Department of Radiation Oncology, William Beaumont Health System, Royal Oak, MI, USA. ${ }^{3}$ Department of Clinical Medicine, Department of Oncology, Aarhus University, Aarhus, Denmark.

\section{Received: 23 February 2015 Accepted: 25 June 2015} Published online: 02 September 2015

\section{References}

1. Burmeister J, McDermott P. Effect of MLC leaf width on the planning and delivery of SMLC IMRT using the CORVUS inverse treatment planning system. Med Phys. 2004;31(12):3187-93.

2. Wu Q, Wang Z, Kirkpatrick J, Chang Z. Impact of collimator leaf width and treatment technique on stereotactic radiosurgery and radiotherapy plans for intra-and extracranial lesions. Radiat Oncol. 2009;4:3.

3. Dvorak P, Georg D, Bogner J. Impact of IMRT and leaf width on stereotactic body radiotherapy of liver and lung lesions. Int J Radiat Oncol Biol Phys. 2005;61(5):1572-81.

4. Monk J, Perks J, Doughty D, Plowman P. Comparison of a micro-multilea collimator with a 5-mm-leaf-width collimator for intracranial stereotactic radiotherapy. Int J Radiat Oncol Biol Phys. 2003;57(5):1443-9.

5. Topolnjak R. Influence of the linac design on intensity-modulated radiotherapy of head-and-neck plans. Phys Med Biol. 2007:52(1):169-82.

6. Chae S-M, Lee GW, Son SH. The effect of multileaf collimator leaf width on the radiosurgery planning for spine lesion treatment in terms of the modulated techniques and target complexity. Radiat Oncol. 2014;9(1):72.

7. Bortfeld T, Oelfke U, Nill S. What is the optimum leaf width of a multileaf collimator? Med Phys. 2000;27(11):2494-502

8. Low DA, Sohn JW, Klein EE, Markman J. Characterization of a commercial multileaf collimator used for intensity modulated radiation therapy. Med Phys. 2001;28(5):752-6.

9. Ramsey C, Spencer K. Leaf position error during conformal dynamic arc and intensity modulated arc treatments. Med Phys. 2001:28(1):67-72.

10. Wijesooriya $K$, Bartee C, Siebers J. Determination of maximum leaf velocity and acceleration of a dynamic multileaf collimator: Implications for 4D radiotherapy. Med Phys. 2005;32(4):932-41.

11. Budgell G, Mott J. Requirements for leaf position accuracy for dynamic multileaf collimation. Phys Med Biol. 2000;45(5):1211-27.

12. Vorwerk $H$, Wagner $D$, Hess $C$. Impact of different leaf velocities and dose rates on the number of monitor units and the dosevolume-histograms using intensity modulated radiotherapy with sliding-window technique. Radiat Oncol. 2008;3:31

13. Webb S. Historical perspective on IMRT. In: Intensity-Modulated Radiation Therapy - The State of the Art. Bristol, UK: Institute of Physics Publishing; 2003. p. 1-23.

14. Tacke MB, Nill S, Häring P, Oelfke U. 6 MV dosimetric characterization of the 160 MLC $^{\text {TM }}$, the new Siemens multileaf collimator. Med Phys. 2008;35(5):1634.

15. van Kesteren $Z$, Janssen TM, Damen E, van Vliet-Vroegindeweij C. The dosimetric impact of leaf interdigitation and leaf width on VMAT treatment planning in Pinnacle: comparing Pareto fronts. Phys Med Biol. 2012:57(10):2943-52

16. Lafond C, Chajon E, Devillers A, Louvel G, Toublanc S, Olivier M, et al. Impact of MLC leaf width on volumetric-modulated arc therapy planning for head and neck cancers. J Appl Clin Med Phys. 2013;14(6):4074

17. Cosgrove VP, Thomas MDR, Weston SJ, Thompson MG, Reyneart N, Evans CJ, et al. Physical Characterization of a New Concept Design of an Elekta Radiation Head with Integrated 160-leaf Multi-leaf Collimator. Int J Radiat Oncol Biol Phys. 2009:75(3):S722-723.

18. Alber M. A Concept for the Optimization of Radiotherapy. PhD thesis. Tübingen: Fakultät für Physik der Eberhard-Karls-Universität zu Tübingen; 2000

19. Alber M, Birkner M, Laub W, Nüsslin F. Hyperion: an integrated IMRT planning tool. In: Proc. XIIIth Int. Conf. onthe Use of Computers in Radiation Therapy. Berlin, Heidelberg: Springer Berlin Heidelberg; 2000.

20. Alber $M$, Reemtsen $R$. Intensity modulated radiotherapy treatment planning by use of a barrier-penalty multiplier method. Optim Methods Softw. 2007;22(3):391-411.
21. Alber M, Nüsslin F. An objective function for radiation treatmen optimization based on local biological measures. Phys Med Biol. 1999:444:479-93

22. Alber M. Normal tissue dose-effect models in biological dose optimisation. Z Med Phys. 2008;18(2):102-10

23. Jeleń $U$, Söhn M, Alber M. A finite size pencil beam for IMRT dose optimization. Phys Med Biol. 2005;50(8):1747-66.

24. Jeleń U, Alber M. A finite size pencil beam algorithm for IMRT dose optimization: density corrections. Phys Med Biol. 2007;52(3):617-33.

25. Unkelbach J, Bortfeld T, Craft D, Alber M, Bangert M, Bokrantz R, et al. Optimization approaches to volumetric modulated arc therapy planning. Med Phys. 2015;42(3):1367-77.

26. Fippel M. Fast Monte Carlo dose calculation for photon beams based on the VMC electron algorithm. Med Phys. 1999;26(8):1466.

27. Bedford JL, Thomas MDR, Smyth G. Beam modeling and VMAT performance with the Agility 160-leaf multileaf collimator. J Appl Clin Med Phys. 2013;14(2):4136

28. Budach W, Bölke E, Homey B. Severe cutaneous reaction during radiation therapy with concurrent cetuximab. N Engl J Med. 2007:357(5):514-5.

29. Kantz S, Ganswindt U, Alber M, Söhn M. Impact of MLC properties and IMRT Technique in Meningeoma and Head and Neck. Int J Radiat Oncol Biol Phys. 2012;84(3):861

30. Paddick I. A simple scoring ratio to index the conformity of radiosurgical treatment plans: technical note. J Neurosurg. 2000;93(3):219-22.

31. Guckenberger M, Richter A, Krieger T. Is a single arc sufficient in volumetricmodulated arc therapy (VMAT) for complex-shaped target volumes? Radiother Oncol. 2009;93(2):259-65.

32. Mancini B, Wilkinson J. Intensity-modulated radiation therapy or volumetricmodulated arc therapy to reduce alopecia, xerostomia, and otitis after whole brain radiation therapy for brain metastases: a. J Radiat Oncol. 2013;2:177-83.

33. Bertelsen A, Hansen C. Single arc volumetric modulated arc therapy of head and neck cancer. Radiother Oncol. 2010:95(2):142-8.

34. Leal A, Sánchez-Doblado F, Arráns R. MLC leaf width impact on the clinical dose distribution: a Monte Carlo approach. Int J Radiat Oncol Biol Phys. 2004;59(5):1548-59.

35. Nill S, Tücking T, Münter M, Oelfke U. Intensity modulated radiation therapy with multileaf collimators of different leaf widths: a comparison of achievable dose distributions. Radiother Oncol. 2005;75(1):106-11.

36. Wang S, Gong Y, Xu Q, Bai S, Lu Y, Jiang Q, et al. Impacts of multileaf collimators leaf width on intensity-modulated radiotherapy planning for nasopharyngeal carcinoma: analysis of two commercial elekta devices. Med Dosim. 2011;36(2):153-9.

37. Zwicker F, Hauswald H, Nill S. New multileaf collimator with a leaf width of $5 \mathrm{~mm}$ improves plan quality compared to $10 \mathrm{~mm}$ in step-and-shoot IMRT of HNC using integrated boost procedure. Strahlentherapie und Onkol. 2010;186(6):334-43.

38. Wolff D, Stieler F, Welzel G. Volumetric modulated arc therapy (VMAT) vs, serial tomotherapy, step-and-shoot IMRT and 3D-conformal RT for treatment of prostate cancer. Radiother Oncol. 2009;93(2):226-33.

39. Stieler F, Wolff D, Schmid H, Welzel G. A comparison of several modulated radiotherapy techniques for head and neck cancer and dosimetric validation of VMAT. Radiother Oncol. 2011;101(3):388-93.

40. Vanetti E, Clivio A, Nicolini G. Volumetric modulated arc radiotherapy for carcinomas of the oro-pharynx, hypo-pharynx and larynx: a treatment planning comparison with fixed field IMRT. Radiother Oncol. 2009;92:111-7.

41. Fogliata A, Clivio A, Nicolini G. Intensity modulation with photons for benign intracranial tumours: a planning comparison of volumetric single arc, helical arc and fixed gantry techniques. Radiother Oncol. 2008:89:254-62.

42. Rao M, Yang W, Chen F, Sheng K, Ye J. Comparison of Elekta VMAT with helical tomotherapy and fixed field IMRT: plan quality, delivery efficiency and accuracy. Med Phys. 2010;37(3):1350-9.

43. Wiezorek T, Brachwitz T, Georg D. Rotational IMRT techniques compared to fixed gantry IMRT and tomotherapy: multi-institutional planning study for head-and-neck cases. Radiat Oncol. 2011;6(1):20.

44. Shang J, Kong W, Wang Y-Y, Ding Z, Yan G, Zhe H. VMAT planning study in rectal cancer patients. Radiat Oncol. 2014;9(1):219.

45. Jeong Y, Lee S, Kwak J, Cho I, Yoon SM, Kim JH, et al. A dosimetric comparison of volumetric modulated arc therapy (VMAT) and non-coplanar intensity modulated radiotherapy (IMRT) for nasal cavity and paranasal sinus cancer. Radiat Oncol. 2014;9(1):193. 
46. Verbakel W, Cuijpers J. Volumetric intensity-modulated arc therapy vs. conventional IMRT in head-and-neck cancer: a comparative planning and dosimetric study. Int J Radiat Oncol Biol Phys. 2009;74(1):252-9.

47. Peters $\mathrm{S}$, Schiefer $\mathrm{H}$, Plasswilm L. A treatment planning study comparing Elekta VMAT and fixed field IMRT using the varian treatment planning system eclipse. Radiat Oncol. 2014;9(1):153.

Submit your next manuscript to BioMed Central and take full advantage of:

- Convenient online submission

- Thorough peer review

- No space constraints or color figure charges

- Immediate publication on acceptance

- Inclusion in PubMed, CAS, Scopus and Google Scholar

- Research which is freely available for redistribution 